\title{
Mobile femtocell utilisation in LTE vehicular environment: Vehicular penetration loss elimination and performance enhancement
}

\author{
Rand Raheem*, Aboubaker Lasebae, Mahdi Aiash, Jonathan Loo, Robert H Colson \\ School of Science E Technology, Middlesex University, The Burrough, NW4 4BT, London, United Kingdom
}

\begin{abstract}
A B S T R A C T
Mobile computing is fast becoming a vital part of everyday life in which User Equipment (UE) demand being reachable anywhere and at anytime, as they spend much time travelling from one place to another, often by trains or buses. The ultimate aim of passengers is the ability to be connected to the Internet while they are moving from one place to another with their mobile devices. Providing indoor coverage on trains and buses directly with outdoor Base Stations (BSs) may not be a good solution due to the high density of use and path losses in the LTE network. This limitation can result in poor signal quality inside the train, and offering broadband services is not always possible. Clearly improvement to broadband access on buses and trains could be achieved by installing more BSs close to railway and bus routes and terminals. However, this solution is not ideal for the Internet Service Providers (ISPs) due to the high investment needed to deploy many more BSs. In addition, such a solution will introduce additional complexity by increasing the number of Handovers (HOs). This issue has focused the research community effort on developing solutions that take advantage of the existing wireless infrastructure without increasing the number of BSs. One method being considered is the development of more efficient methods and technologies to manage the UE's mobility in seamless ways. In this paper we propose adoption of Mobile Femtocell (Mobile-Femto) technology as a solution to mitigate the Vehicular Penetration Loss (VPL) and Path Loss, with consequent improvement to the vehicular UE's performance in LTE networks. Our results, using a Matlab simulation model, showed a noticeable improvement in the achieved Ergodic capacity by 5\% under a VPL of $40 \mathrm{~dB}$ while $90 \%$ of vehicular UEs spectral efficiency has improved by $1.3 \mathrm{~b} / \mathrm{cu}$ under a VPL of $25 \mathrm{~dB}$. In addition, $80 \%$ of vehicular UEs have improved their throughput and SINR by $300 \mathrm{~kb} / \mathrm{s}$ and $4 \mathrm{~dB}$ respectively after implementing the Mobile-Femto into the Macrocell in LTE networks.
\end{abstract}

Keywords:

Mobile femtocell

Penetration loss

Vehicular user equipment

Intelligent transportation system

Physical layer

Internet communication

\section{Introduction}

In mobile and ubiquitous networks, it is desirable that UEs do not experience fluctuations in the service quality when they are moving from one place to another. In this sense, Mobile-Femto architecture has been introduced to improve the $3 \mathrm{G}$ and $4 \mathrm{G}$ connectivity inside the bus environment to support mobility between vehicular UEs and the core network in LTE networks [15]. The main advantage of implementing the Mobile-Femto is the ability of this small cell to move around and dynamically change its connection with the operator's core network. This Mobile-Femto concept can be seen as a practical implementation of the moving networks that

\footnotetext{
* Corresponding author.

E-mail addresses: R.Raheem@mdx.ac.uk (R. Raheem), A.Lasebae@mdx.ac.uk (A. Lasebae), M.Aiash@mdx.ac.uk (M. Aiash),J.Loo@mdx.ac.uk (J. Loo), R.Colson@mdx.ac.uk (R.H Colson).
}

can be deployed in public transportation to overcome the high penetration loss and path-loss issues. Therefore, Mobile-Femto can reduce the impact of vehicular environment on UEs SNIR, throughput, spectral efficiency and Ergodic capacity.

Added to this, UEs inside public transportation may initiate multiple HOs and this may cause a significant increase in the signalling load with a resulting drop in network connections. This has led us to look at Mobile-Femtos as a solution to minimise the signalling load, the number of dropped packets and the number of HOs [20].

Thus, Fig. 1 represents the fixed and mobile Femtocells that could be either inside buildings, on streets or public transportation like trams and buses.

The Mobile-Femto architecture in LTE system is as shown in Fig. 2. This figure shows that there are three types of links that have been utilised to differentiate between eNB \& Mobile-Femto, Mobile-Femto \& UE, and eNB \& UE links which are the backhaul link, the access link and the direct link respectively. 


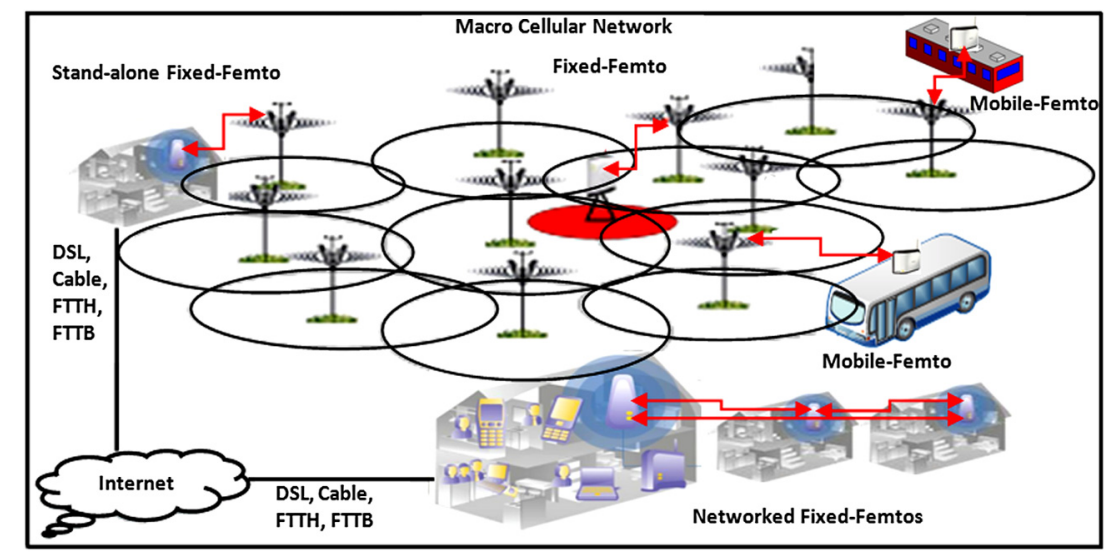

Fig. 1. Fixed and Mobile Femtocell technology [29].

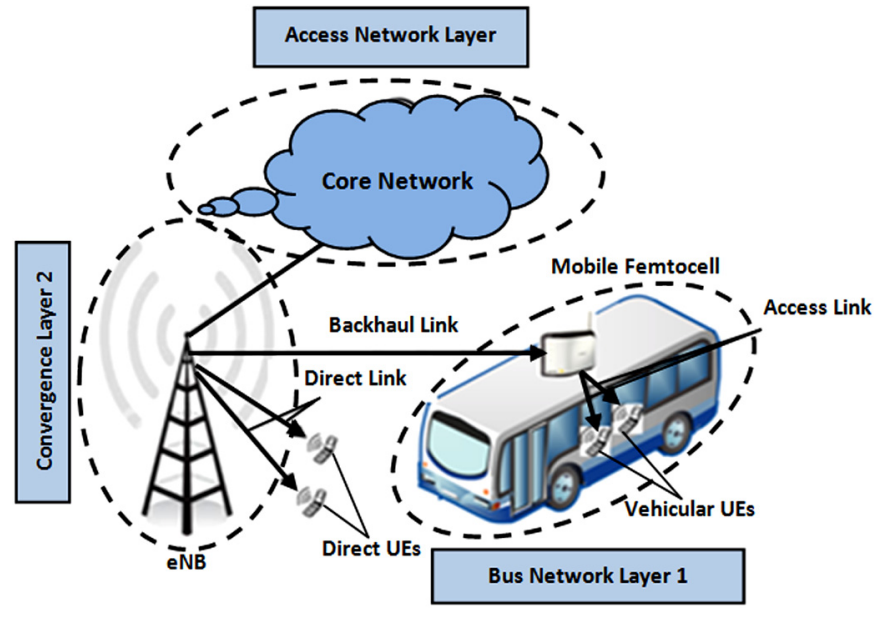

Fig. 2. Mobile Femtocell architecture with its layering system.

Hence, the Mobile-Femto architecture relies on three different designed layers as the following:

1. The Bus Network Layer (BNL) consists of the Mobile-Femto in the bus and all the vehicular UEs (passengers) attached to this Femtocell.

2. Convergence Layer (CL) aggregates the traffic sent by the Mobile-Femtos in the BNLs via the backhaul links and forwards it to the Internet. The eNBs or the mother BSs enable connectivity for the Mobile-Femto technology that is installed in the bus with the outside environment.

3. The Access Network Layer (ANL) comprises the outdoor wireless technology that is available along the bus paths, e.g. LTE technology. Thus, the ANL is the LTE core network and it is the decision maker ahead of the eNB in the LTE systems.

The described Mobile Femtocell avoids the multiple HO procedures since a single $\mathrm{HO}$ is required between the vehicular UE and the serving Femtocell in the bus, instead of performing many HO procedures for each UE. It also improves the mobile devices battery life due to the short distance between those mobile devices and the serving Femtocell that is installed in public transportation. In addition, it makes a better use of the coverage area, because of the use of a single omnidirectional antenna that gives equal signal strength distribution. The most important thing about implementing those open access small BSs inside buses [27] is the ability of these BSs to eliminate the VPL, path-loss and fading issues that vehicular UEs are exposed to. This can improve the efficiency of the vehicular UEs SINR, throughput, spectral efficiency and system ca- pacity. However, the deployment of these small cells has brought many mobility and interference challenges that have all been discussed in our previous works [20] and [26]. In [20] we have discussed the impact of deploying the Mobile-Femto technology on the unnecessary number of HOs, dropped and blocked call probabilities together with the outage probability. On the other hand, in [26] we have presented the raised interference issue that is caused by the deployment of these small cells and proposed suitable solutions such as optimise the cell planning technique, control the transmission power and deploy the Fractional Frequency Reuse scheme. The previous techniques have efficiently mitigated the interference issue which has been noticed through the improvement of the achieved SINR and throughput.

However, in this paper we are more concerned with the impact of deploying these small fixed and vehicular cells on the performance of vehicular UEs. It is very important to evaluate the benefits that these small cells have brought in terms of throughput, SINR, Ergodic capacity and spectral efficiency.

\section{Related work}

In LTE networks, the indoor coverage can be severely degraded by penetration losses through the walls of buildings. If the BS is outdoor but the mobile is indoor, then the penetrations losses typically reduce the received signal power by 10 to 20 decibels (a factor of 10 to 100 ), which can greatly reduce the indoor coverage [30]. This is one of the reasons behind the progressive introduction of Femtocells [19]. It is worth noting that a similar limitation applies to vehicular UEs. These UEs experience high VPL since there is a barrier - the vehicle's chassis - between the UEs and the outdoor BSs, and this reduces the strength of the transmitted and received signals. Other issues have been stated in [2]. This study showed that high-speed trains can be a fruitful environment for mobile services as users are concentrated in relatively small areas. In the train environment, the trains' paths are always known and the railway environment itself has large tunnels, wide cuttings and curves. However, several issues arise in such an environment like fading, Doppler, transients, and penetration loss into carriages, as well as special situations such as cuttings and tunnels. This creates a problem with the operation of the physical layer as this may affect the link between the UE and the outdoor serving BS that causes performance degradation at the highest train speeds.

While in [12], the authors present a series of VPL measurements performed in $800 \mathrm{MHz}$-frequency band. These measurements were conducted for three different vehicle types, mini-van, full size car and sport car with different types of environments e.g. urban or suburban. The statistical properties of the VPL have been examined in order to determine the benchmark parameters to be 
used in the design of wireless communication systems. While the achieved results showed that the vehicle's chassis, speed and distance from the serving BS play very effective roles in term of the quality of the transmitted signal. Hence, this study has made it clear why the vehicular UEs in trains suffer from the worst link connection with the outdoor BSs.

Vehicular UEs are most affected by high penetration losses due to the signal strength fluctuation and radio link failures between the vehicular UEs and the outdoor BSs and clearly the mobility aspect is an adverse factor in this situation. The signal quality inside vehicular environment is very poor due to the high VPL [28], path-loss and fading. However, poor signal means poor SINR, as in wireless communication the SINR is the key indicator of signal quality in wireless connections. Therefore in order to improve the SINR inside vehicular environments, indoor coverage can be deployed with effective results, as study [6] shows. In this study, the authors discuss the ability of improving the QoS of vehicular UEs and solving the issues behind the low SINR by deploying mobile Femtocells in the Macrocell. Another study however has discussed the cell-edge vehicular UEs who suffer from low SINR and performance degradation in general [4]. The authors have considered the feasibility of Decode and Forward (DF) Relay nodes from the 3GPP LTE-Advanced perspective as an attractive solution to solve the SINR reduction. The proposed solution is based on finding the relation between the Relay node transmission power, the ratio between the number of BSs and Relay nodes and the performance of the system. The achieved results showed a good performance in term of the signal strength after the deployment of the Relay nodes.

On the other hand, other factors play important role in the vehicular UEs performance include the spectrum/spectral efficiency or the BW efficiency. The spectral efficiency utilisation is more affected by the UE's mobility and speed. The authors in [9] have discussed the ability of improving the spectrum efficiency using the mobile Femtocell technology. This study stated that the spectral efficiency of mobile Femtocells UE can be improved with the use of two resource partitioning schemes, orthogonal and nonorthogonal. While [11], has discussed the problem of resource allocation in a cellular network with the deployment of mobile Femtocells. This study showed that the speed and path information of the mobile Femtocells have been used to determine the interference correlations between different Femtocells at different time instants, and represent them as a time interval dependent interference graph.

Other studies like [13] and [3] have shown that the Radio Resource Management (RRM) model that is used in LTE systems is responsible for the spectrum resource, channel allocation, transmission power and modulation schemes. These studies have proposed different resource allocation schemes to allocate resources between the Macrocell and the Femtocell over the shared spectrum. In these studies, the Femtocells try to learn the resource usage pattern of Macrocells based on their synchronisation, and adjust the resource block pattern based on the interference. Whenever, the Femtocell finds a free slot from Macrocell, it allocates the free resource block to Femtocell's UEs. This is applicable only when there is less traffic, which may generate high interference in the case of high traffic loads.

In another study, authors in [14] have proposed an alternative method for the mobile environment, which is the use of multioperator mobile Relay nodes for cellular networks on buses and trains. This study has enabled an improvement in the spectral efficiency because an antenna with higher gain than that of UE has been installed in the Relay node.

Added to this, the network throughput is more affected by the UE's mobility and speed. The speed has the biggest impact on the UE's quality of connection. Accordingly, many researchers have considered this issue as an area of interest. In [16], the authors presented system level simulation results for a cooperative moving Relay node system deployed on a High Speed Train (HST). This provided enhanced cellular coverage to UEs in public transportation, particularly HSTs, where modern construction materials and techniques cause high VPL. This study showed that mobile Relay nodes utilising antenna arrays on the exterior and interior of the train are a promising method of overcoming this VPL in order to provide onboard UEs with improved services. The achieved results showed a slight improvement in the achieved throughput of onboard UEs when compared to direct transmission of the vehicular UEs. Another study has considered the mobile Relays as a solution to improve the vehicular UEs throughput as in [17]. Here, the authors have considered the mobile Relay node to be deployed on public transportation to serve vehicular UEs in order to reduce the impact of the VPL and improve the UEs throughput. However, both of the previous studies were limited by the number of served UEs (i.e. max five UEs), and the adopted coverage areas. Another issue raised by the second study is the random movement of the mobile Relay which accentuates the interference problems.

Many previous studies (e.g. [16] and [14]) have shown that the increased demand for using the new multimedia services and features of today's Smart-Phones in vehicular environment have been considered as a drawback in current networks. This is because vehicular UEs may not be able to connect to the network directly without the use of an efficient technology to cover the network holes and improve the vehicular UEs performance. Thus, several challenges need to be addressed when considering the deployment of Mobile-Femto technology. These include the resource distribution between the Macrocell and the Mobile-Femto, the UEs scheduling process, the vehicular UEs spectral efficiency, throughput, SINR, and link Ergodic capacity to accommodate the increased amount of transmitted data. An additional concern is to reduce the effect of path-loss and VPL in the vehicular environments, and to improve the performance of those vehicular UEs.

Therefore we have considered the potential of deploying mobile BSs in the Macrocell to enhance the vehicular UEs SINR, throughput and spectral efficiency. In the following sections we model, evaluate and compare the vehicular UEs performance before and after deploying the Fixed-Femto and Mobile-Femto into the LTE Macrocell.

\section{System model}

The communication process between the eNB and the Femtocell and between the Femtocell and the UE in LTE system occurs in the following manner. The eNB gathers the Channel State Information (CSI) from all UEs and Fixed-Femtos/Mobile-Femtos in the Macrocell. Likewise, the UEs within the Fixed-Femto/Mobile-Femto coverage will feedback this information only to the Fixed-Femto/MobileFemto. In the transmission process, the eNB transmits the data to the selected Fixed-Femto/Mobile-Femto via the backhaul link and then the Fixed-Femto/Mobile-Femto will fully decode the data, buffer it and retransmit it to its UE via the access link. Hence, Fig. 3 depicts the considered eNB which has a fixed coverage of $D$ meters depending on the chosen transmission power, while one vehicle (bus) is moving along the highway with a number of UEs inside it. It has been assumed that both the Fixed-Femto and the Mobile-Femto employ dual-hop transmission where the eNB transmits to a vehicular UE via the Fixed-Femto/Mobile-Femto and vice versa. Additionally, $d$ meters is the distance between the eNB and the Fixed-Femto while $x$ is the distance between the eNB and the vehicular UE. 


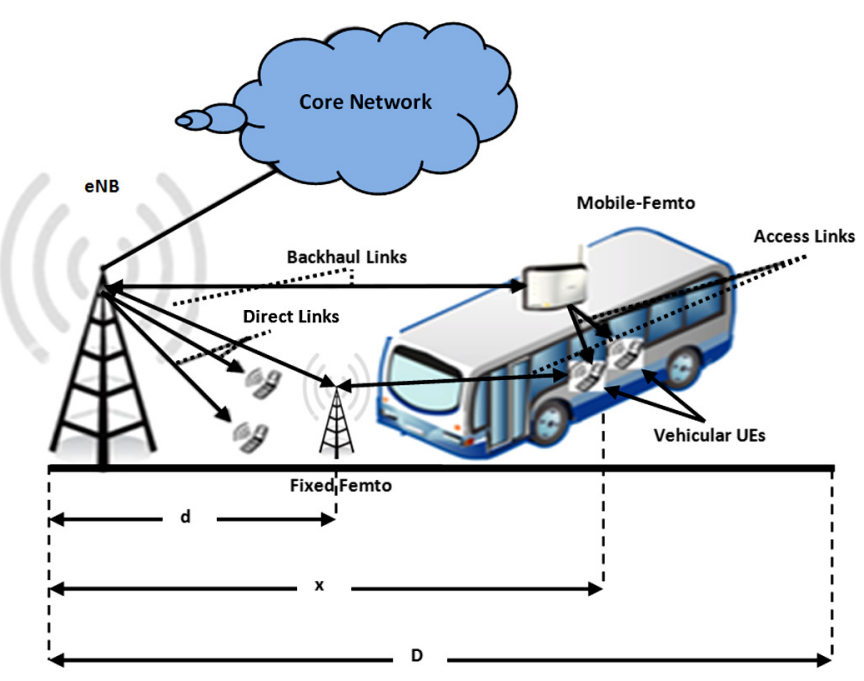

Fig. 3. eNB, fixed \& Mobile Femtos system model architecture.

It can be shown that, the received signal-to-noise ratio (SNR) at the receiver $\left(R_{X}\right)$ side can be given by

$S N R_{R_{X}}=\frac{P_{x}|h|^{2} P L(L) \varepsilon}{P_{\text {noise }}}$

where the $h$ represents the channel coefficient and PL has been used to model the path-loss when the receiver $R_{X}$ is at distance $L$ away from the transmitter $T_{x}$. The $P_{x}$ is the average transmission power at the transmitter $T_{\chi}$. Moreover, $\varepsilon$ is the VPL and $P_{\text {noise }}$ represents the noise power.

As shown earlier in Fig. 3, the vehicular UEs is at distance $x$ away from the eNB. Thus, according to Shannon equation the capacities of the backhaul and access links can be given as $C_{\text {backhaul }}=$ $B W_{\text {eNB-femto }} \log _{2}\left(1+S N R_{\text {femtocell }}\right)$ and $C_{\text {access }}=B W_{\text {femto-UE }} \log _{2}(1+$ $\left.S N R_{U E}\right)$ respectively [7], where

$S N R_{e N B-U E}=\frac{P_{x}^{e N B}\left|h_{1}\right|^{2} P L(x) \varepsilon}{P_{\text {noise }}}$

and the SNR of the Fixed-Femto assisted-transmission can be given as

$S N R_{F F B S-U E}=\frac{P_{x}^{F F B S}\left|h_{2}\right|^{2} P L(x-d) \varepsilon}{P_{\text {noise }}}$

On the other hand, the distance between the transmitter $T_{X}$ of the vehicular UE and the Femtocell that is allocated in the same bus (Mobile-Femto), is less than 5 meters at most. As a result, a LOS access link and a constant loss $C_{\text {loss }}$ have been assumed. The constant loss $C_{\text {loss }}$ is the same as the Constant Path-Loss, which is a free space loss when there is no obstacle against the transmitted and received signals. Hence, the SNR of the Mobile-Femto assistedtransmission is given by

$S N R_{M F B S-U E}=\frac{P_{x}^{M F B S} C_{\text {loss }}}{P_{\text {noise }}}$

Here, the $P_{X}^{e N B}, P_{X}^{F F B S}$ and $P_{x}^{M F B S}$ denote the average transmission power of the eNB, Fixed-Femto and Mobile-Femto while $h_{1}$ denotes the channel coefficient of the direct link and $h_{2}$ denotes the channel coefficient of the access link in the Fixed-Femto assisted transmission. The channel coefficient of the Mobile-Femto assistedtransmission has been assumed to be unity (equal 1) due to the very short distance between the UE and the installed Femtocell in the same bus as well as the LOS access link.

After presenting the SNR of the eNB, Fixed-Femto and MobileFemto assisted transmissions, now it becomes necessary to state the Ergodic capacity of the backhaul, direct and access links. The backhaul links between the eNB-Fixed Femtos and the eNB-Mobile Femtos are assumed to be NLOS outdoor links. Therefore, the backhaul link Ergodic capacity between the eNB and the Fixed-Femto at distance $d$ can be given by

$$
C_{\text {backhaul }}(e N B-F F B S)=B W_{e N B-F F B S} \log _{2}\left(1+\frac{P_{x}^{e N B}\left|h_{1}\right|^{2} P L(d)}{P_{\text {noise }}}\right)
$$

While the backhaul link Ergodic capacity between the eNB and the Mobile-Femto at distance $x$ can be given by

$C_{\text {backhaul }}(e N B-M F B S)=B W_{e N B-M F B S} \log _{2}\left(1+\frac{P_{x}^{e N B}\left|h_{1}\right|^{2} P L(x)}{P_{\text {noise }}}\right)$

It should be noted that in the backhaul link Ergodic capacity between the eNB and the Mobile-Femto there is a small channel gain. This results from the high path-loss between the Mobile-Femto and the eNB, as well as the NLOS backhaul link.

The Ergodic $C_{\text {direct }}(e N B-U E)$ can be equated with Ergodic $C_{\text {backhaul }}(e N B-M F B S)$ in equation (6), since the direct link between the eNB and the vehicular UEs is a NLOS link, and the distance between the eNB and the UE is the same as the distance between the eNB and the Mobile-Femto.

Hence, the access link Ergodic capacity between the FixedFemto and the vehicular UE at distance $x-d$ can be derived and given as

$C_{\text {access }}(F F B S-U E)=B W_{\text {FFBS-UE }} \log _{2}\left(1+\frac{P_{x}^{F F B S}\left|h_{2}\right|^{2} P L(x-d) \varepsilon}{P_{\text {noise }}}\right)$

While the access link Ergodic capacity between the Mobile-Femto and the vehicular UE is a special case scenario as the VPL is not exist in this case. This is because there are no barriers between the UEs and the serving BS so nothing resists the signal from reaching the UEs without losses. Therefore, the link capacity can be given by

$C_{\text {access }}(M F B S-U E)=B W_{M F B S-U E} \log _{2}\left(1+\frac{P_{x}^{M F B S} C_{\text {loss }}}{P_{\text {noise }}}\right)$

Here the $B W_{e N B-F F B S}$ and $B W_{e N B-M F B S}$ represent the bandwidth of backhaul links between eNB-FixedFemto and eNB-MobileFemto while $B W_{F F B S-U E}$ and $B W_{M F B S-U E}$ represent the bandwidth of access links between FixedFemto-UE and MobileFemto-UE respectively.

After clarifying the communication links between the eNBs, Fixed-Femtos and Mobile-Femtos, it is significant now to go further and discuss the UEs scheduling process and resource allocation scheme in these BSs. A multiuser scheduling scheme is assumed where the Macrocell UEs, the Mobile-Femtos and the Fixed-Femtos UEs are served over $k$ Physical Resource Blocks (PRBs), indexed by $k=1, \ldots, K$. The Fixed and Mobile Femtos are scheduled over a dedicated time-frequency zone in such a set of Fixed-Femtos and Mobile-Femtos that are selected according to scheduling criterion. Fig. 4 shows the scheduling mechanism in term of eNB, MobileFemtos and Fixed-Femtos UEs.

The eNB is responsible for scheduling all the links of the network, Femtocells' links and UEs' links. The Femtocell nodes only forward the received data and signalling from/to the eNB without any scheduling. The scheduler in the eNB should take into account the limitation of the Control Channel Elements (CCEs) when allocating the PRBs to the UEs in both directions Uplink and Downlink (UL and DL). Therefore, the UEs scheduling has two successive scheduling decisions; the candidates selection followed by frequency domain resources allocation to assign the PRBs among the selected UEs. It is to be mentioned that, the candidates' selection can be either UEs or Femtocells who need to be scheduled in the Macrocell. The eNB will schedule the Mobile-Femtos like any 


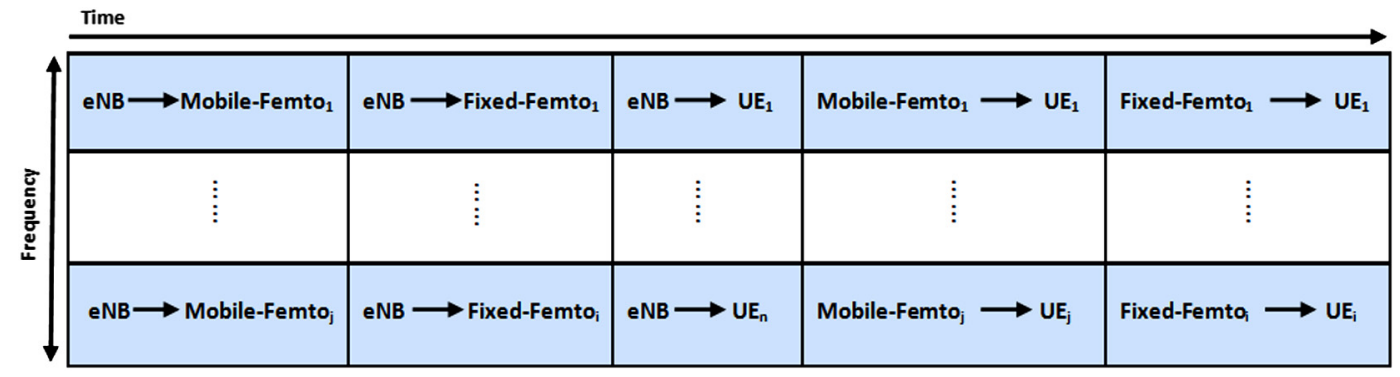

Fig. 4. Time sharing strategy for fixed and Mobile Femtos in LTE.

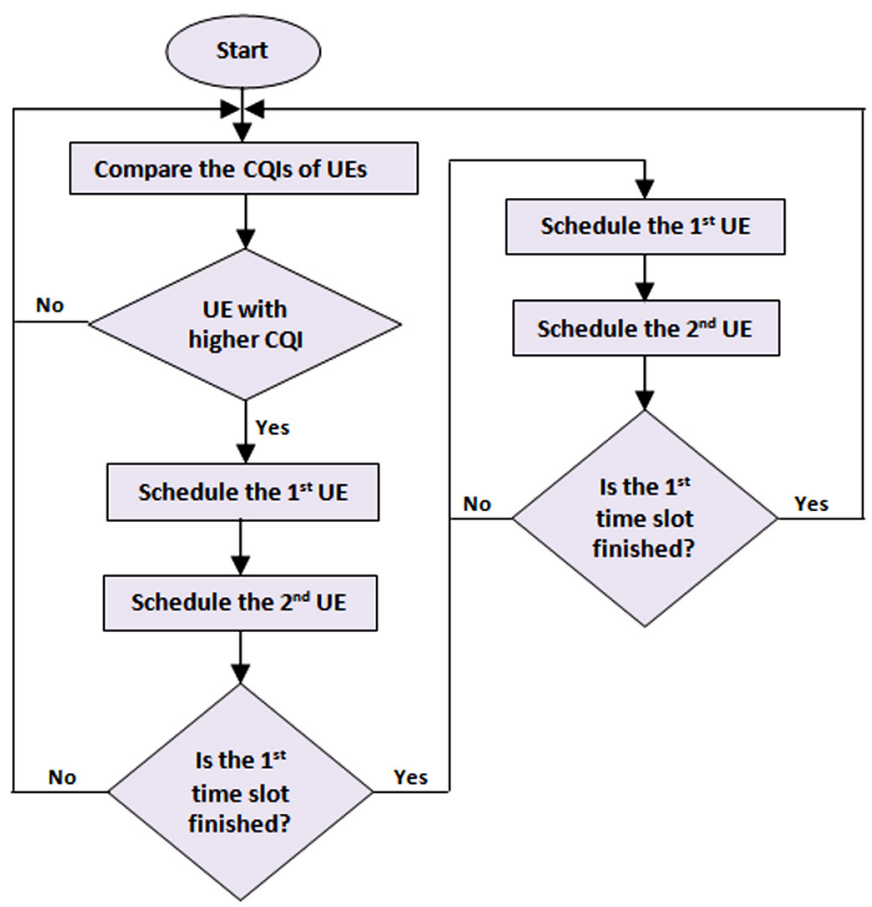

Fig. 5. Proportional fair scheduler for UEs in LTE network.

other UEs but of course, more PRBs will be allocated to those access points than normal UEs need. Hence, the scheduling process occurs as the following;

1. First, the time domain scheduler will prioritise the UEs based on a given priority criterion e.g. proportional fair.

2. Second, it selects only Macro UEs or Mobile-Femtos/FixedFemtos with highest scheduling priority taking into account the total Control Channel Elements (CCEs) constraints as well as the number of available PRBs. This can be defined as $(N$ UEs $n \leq N_{\max }$ ), ( $J$ Mobile-Femtos $j \leq J_{\max }$ ) or ( $I$ Fixed-Femtos $\left.i \leq I_{\max }\right)$, where $n \in N=\{1, \ldots, N\}$ denotes the set of UEs who communicate directly with the eNB (Macrocell UEs). While $N_{j}$ refers to a group of UEs within a Mobile-Femto $j$ and $N_{i}$ denotes the group of UEs within a Fixed-Femto $i$, where MobileFemto $j \in J=\{1, \ldots, J\}$ and Fixed-Femto $i \in I=\{1, \ldots, I\}$.

This paper considers the Proportional Fair (PF) scheduling policies. This type of scheduling refers to the amount of resources allocated within a given time window to UEs with better channel quality in order to offer high cell throughput as well as fairness satisfactory. The PF scheduling mechanism has been presented by Fig. 5 [8]. This scheduling policy works as the following; firstly, the scheduler sorts the UEs in descending order according to the proportional fair metric and then it picks up only some of the UEs depending on the availability of the CCE, the PRBs and UE's Chan- nel Quality Indicator (CQI). Secondly, the scheduler allocates the PRB $k$ to UE $n$, Mobile-Femto $j$ or Fixed-Femto $i$ according to the following criterion

$n_{k}=\arg \max _{n \in N} \frac{R_{n}(k, t)}{\bar{R}_{n}(t-1)}$

where the $\bar{R}_{n}(t-1)$ denotes the average data rate of UE $n$ before the current scheduling subframe $t$. Thus, $\arg \max _{n \in N} R_{n}(k, t)$, $k=1, \ldots, K$ and $R_{n}(k, t) \propto S N R_{n}$ is the instantaneous achievable rate on PRB $k$ for a user $n$ which can be calculated according to Shannon formula

$R_{n}(k, t)=\frac{B W}{k} \log _{2}(1+\operatorname{SNR}(k, t))$

The average data rate of UE $n$ can be calculated using an exponential average filtering, which will be updated using the following formula:

$\bar{R}_{n}(t)=\left(1-\frac{1}{T}\right) \bar{R}_{n}(t-1)+\frac{1}{T} \sum_{k=1}^{K} R_{n}(k, t) d_{n}(k, t)$

where $T$ is the average window length and $d_{n}(k, t)$ is a binary indicator that is set to 1 if the user $n$ is scheduled on PRB $k$ at time 1 and to 0 otherwise. Bearing in mind that the main concern of this paper is the vehicular environment, vehicular UEs and Femtocells; therefore, the scheduling process may occur differently from the traditional process. This is because the scheduling process here is not only for vehicular UEs but for Femtocells as well. Hence, the availability of BW, and resource blocks, play important roles in the scheduling process of both the UEs and Femtocells. This is because there is a positive correlation between the used BW and the transmitted data rate $\left(R_{n}\right)$. In other words, whenever the used BW is large, the ability of allocating more PRBs to UEs and Femtocells increases. This has a positive influence on the transmitted bit rate and achieved throughput.

Accordingly, the Algorithm 1 represents the UEs and Femtocells PF scheduling scheme in the Macrocell under different traffic loads (low, medium and heavy traffics).

After discussing the scheduling process and PRBs distribution among Macro UEs and Femtocells in the Macrocell, it is important now to discuss the effect of this on the achieved throughput. Thus, based on Shannon Equation, the throughput of direct vehicular UEs at distance $x$ from the eNB can be given by

Throughput of $e N B_{U E}=\log _{2}\left(1+\frac{P_{x}^{e N B}\left|h_{1}\right|^{2} P L(x) \varepsilon}{P_{\text {noise }}}\right) \cdot B W_{e N B}$

whereas, the following represents the throughput of Fixed-Femto vehicular UEs at distance $x-d$ where the VPL plays an important role in this case as below shows

Throughput of $F F B S_{U E}$

$$
=\log _{2}\left(1+\frac{P_{x}^{F F B S}\left|h_{2}\right|^{2} P L(x-d) \varepsilon}{P_{\text {noise }}}\right) \cdot B W_{F F B S}
$$




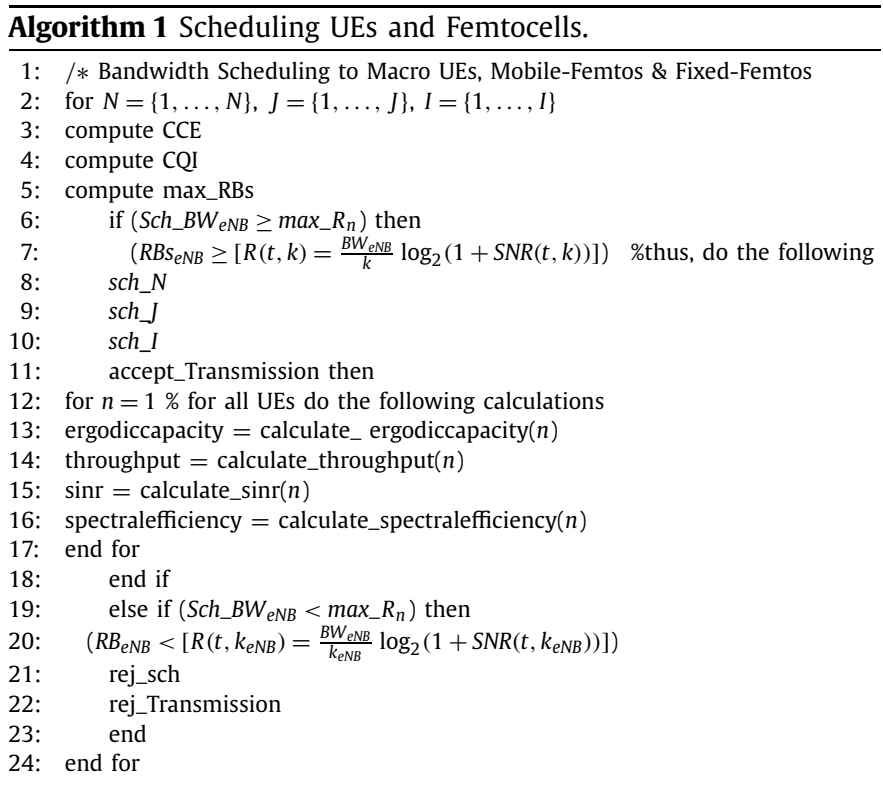

Likewise the following equation represents the Mobile-Femto UEs when the VPL is unity as explained earlier.

Throughput of $M_{F B S_{U E}}=\log _{2}\left(1+\frac{P_{X}^{M F B S} C_{\text {loss }}}{P_{\text {noise }}}\right) \cdot B W_{M F B S}$

where the $B W_{e N B}, B W_{F F B S}$ and $B W_{M F B S}$ is the available bandwidth at the eNB, Fixed-Femto and Mobile-Femto respectively to serve the vehicular UEs. In order to evaluate the impact of deploying Fixed-Femto and Mobile-Femto in the Macrocell to serve the vehicular UEs, the overall vehicular UEs throughput can be calculated before and after deploying the Femtocells into the Macrocell to see the obvious difference as the following formula shows:

Total Throughput $=$ Throughput of $e N B_{U E s}$

$$
\begin{aligned}
& + \text { Throughput of } F F B S_{U E S} \\
& + \text { Throughput of MFBS } \\
& \text { UES }
\end{aligned}
$$

Which can be clearly given by

Total Throughput

$$
\begin{aligned}
= & {\left[\log _{2}\left(1+\frac{P_{x}^{e N B}\left|h_{1}\right|^{2} P L(x) \varepsilon}{P_{\text {noise }}}\right) \cdot B W_{\text {eNB }}\right] } \\
& +\left[\log _{2}\left(1+\frac{P_{x}^{F F B S}\left|h_{2}\right|^{2} P L(x-d) \varepsilon}{P_{\text {noise }}}\right) \cdot B W_{F F B S}\right] \\
& +\left[\log _{2}\left(1+\frac{P_{x}^{M F B S} C_{\text {loss }}}{P_{\text {noise }}}\right) \cdot B W_{\text {MFBS }}\right]
\end{aligned}
$$

However, after deploying the Fixed-Femtos/Mobile-Femtos, the spectrum has to be allocated efficiently among the different links: the backhaul, direct and access links. It is essential therefore, to design an efficient method that improves the spectral efficiency among these three links. It is to be mentioned that the nonorthogonal resource allocation scheme has been applied in which the radio resources are reused by the direct and access links. In contrast the radio resources are orthogonally allocated between the backhaul and the direct links, and between the backhaul and the access links. The non-orthogonal resource allocation scheme indicates that there will be an Inter-Carrier Interference (ICI) to the access and direct UEs due to the simultaneous transmissions from the Mobile-Femto/Fixed-Femto and eNB on the same subchannels. This scheme has several advantages over the orthogonal resource allocation scheme since it improves the resource utilisation as well as giving the flexibility to implement the RRM at the eNB and the Mobile-Femto/Fixed-Femto independently.

All the previous formulas have created the base to calculate the spectral efficiency of vehicular UEs in LTE networks. It is worthwhile to note that the spectral efficiency is the optimum spectrum that is used to provide a large amount of data at a specific BW [21]. In other words, it is defined for each location, as the ratio of throughput to the available BW for a UE under the assumption of one single subscriber in the cell as the following represents

Spectralefficiency $=\frac{\text { Throughput }}{\text { AvailableBW }}$

Moving on from the previous concept of the spectral efficiency to Shannon capacity formula $\left(C=B W \log _{2}\left(1+\frac{S}{N}\right)\right)$, this will help in calculating the maximum (total) spectral efficiency which can be given by [18]

$$
\frac{C}{B W}=\log _{2}\left(1+\frac{S}{N}\right)
$$

where $C$ is the achieved capacity that can be given by bits/sec/Hz, which is the same of the system throughput. Hence, based on (17) and (18) the spectral efficiency can be given by

Spectralefficiency $=\log _{2}\left(1+\frac{S}{N}\right)$

Thus, the spectral efficiency of vehicular UE can be calculated based on the previous SNR formulas for direct and access UEs. The direct vehicular UEs spectral efficiency is given by

Spectralefficiency of $e N B_{U E}=\log _{2}\left(1+\frac{P_{x}^{e N B}\left|h_{1}\right|^{2} P L(x) \varepsilon}{P_{\text {noise }}}\right)$

While the spectral efficiency of the Fixed-Femto vehicular UE at distance $x-d$ can be calculated by the following equation

Spectralefficiency of $F F B S_{U E}=\log _{2}\left(1+\frac{P_{x}^{F F B S}\left|h_{2}\right|^{2} P L(x-d) \varepsilon}{P_{\text {noise }}}\right)$

On the other hand, the spectral efficiency of the Mobile-Femto UE can be given by the following formula

Spectralefficiency of $M F B S_{U E}=\log _{2}\left(1+\frac{P_{x}^{M F B S} C_{\text {loss }}}{P_{\text {noise }}}\right)$

As mentioned earlier, in this case the penetration loss is not exist due to the absence of walls or other obstacles between the vehicular UE and the Mobile-Femto, since both are inside the same vehicle.

After computing the spectral efficiency of the direct and access UEs, it becomes clear that in order to find the total spectral efficiency of the Macrocell, three spectral efficiencies need to be added up. This will help in understanding the impact of deploying the Fixed-Femto/Mobile-Femto into the LTE Macrocell to serve the vehicular UEs.

Total Spectralefficiency

$=$ Spectralefficiency of $e N B_{U E S}+$ Spectralefficiency of $F F B S_{U E S}$

+ Spectralefficiency of MFBS $U E S$

Hence, 


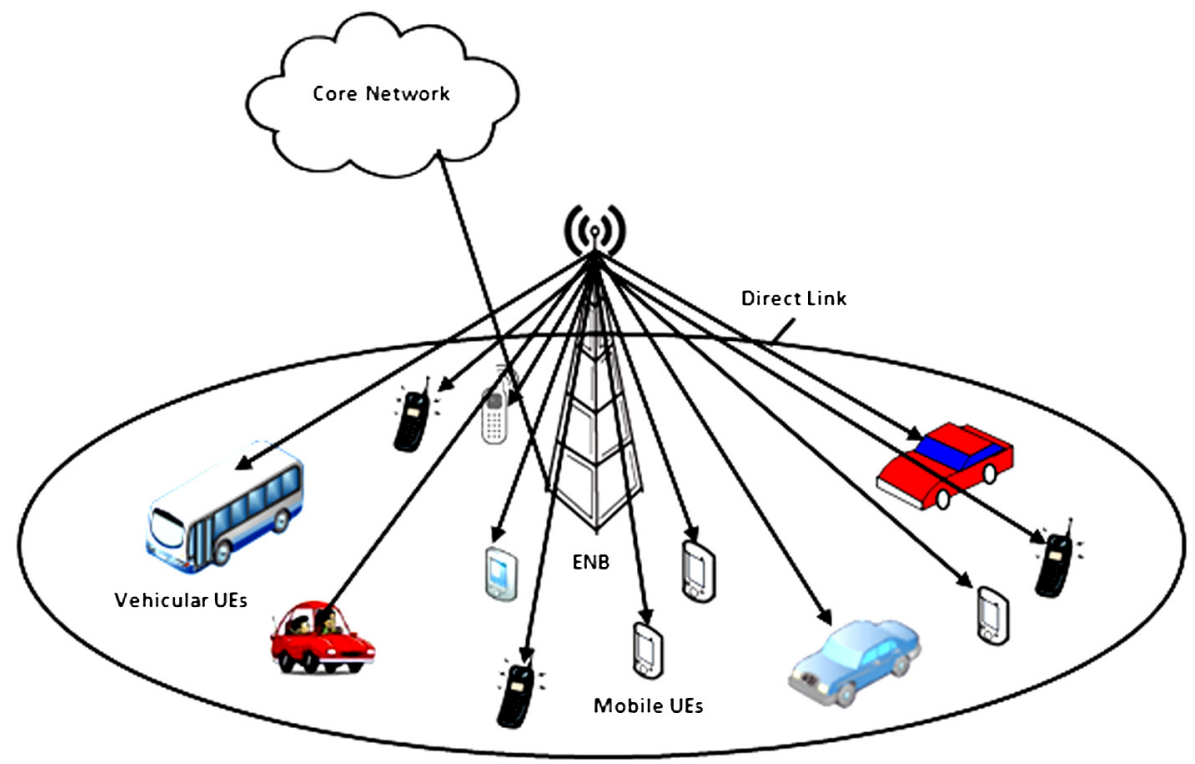

Fig. 6. Vehicular and mobile UEs served by the Macrocell.

Total Spectralefficiency

$$
\begin{aligned}
= & \log _{2}\left(1+\frac{P_{x}^{e N B}\left|h_{1}\right|^{2} P L(x) \varepsilon}{P_{\text {noise }}}\right) \\
& +\log _{2}\left(1+\frac{P_{x}^{F F B S}\left|h_{2}\right|^{2} P L(x-d) \varepsilon}{P_{\text {noise }}}\right) \\
& +\log _{2}\left(1+\frac{P_{x}^{M F B S} C_{\text {loss }}}{P_{\text {noise }}}\right)
\end{aligned}
$$

Now, it is essential to calculate the SINR of Macro and Femtocell UEs as the signal strength of vehicular UEs is the main concern of this work. Based on SINR $=\frac{P_{\text {signal }}}{I+P_{\text {noise }}}$, the received SINR for the Direct vehicular UE $\left(\operatorname{SINR}_{D}\right)$ can be given by

$\operatorname{SINR}_{m(D)}=\frac{P_{x}^{e N B}\left|h_{1}\right|^{2} P L(x) \varepsilon}{\left(I_{M F B S}+I_{F F B S}\right)+P_{\text {noise }}}$

where $I_{F F B S}$ and $I_{M F B S}$ is the ICI from the Fixed-Femto and MobileFemto respectively, $P_{\text {noise }}$ is the noise power, and the $h_{1}$ is the channel coefficient over the direct link. On the other hand, the received SINR for the Access vehicular UE $\left(\operatorname{SINR}_{A}\right)$ in the case of the Fixed-Femto transmission can be calculated according to the following equation

$\operatorname{SINR}_{\text {FFBS (A)UE }}=\frac{P_{x}^{F F B S}\left|h_{2}\right|^{2} P L(x-d) \varepsilon}{\left(I_{e N B}+I_{M F B S}\right)+P_{\text {noise }}}$

where $I_{e N B}$ is the ICI from the eNB and $h_{2}$ is the channel coefficient over the access link between the Fixed-Femto and the vehicular UE. Whilst, the received SINR for the Access vehicular UE $\left(\operatorname{SINR}_{A}\right)$ in the case of the Mobile-Femto can be calculated according to the following formula

$\operatorname{SINR}_{\text {MFemto }(A) U E}=\frac{P_{X}^{M F B S} C_{\text {loss }}}{\left(I_{e N B}+I_{F F B S}\right)+P_{\text {noise }}}$

As mentioned earlier, channel coefficient over the Mobile-Femto access link is unity (equal 1) as the distance between the UEs and the serving Femtocell is very short. Also, the VPL in this case does not exist due to the absences of walls and barriers between the serving Femtocell and UEs. However, the UEs might experience some interference from the eNB and the nearby Fixed-Femtos as it may affect the SINR value.
It is to be mentioned that, the previous interference issues between eNBs and Femtocells, can be mitigated as shown in [26]. This study has shown that using an optimised cell planning technique, control the transmission power and use the Fractional Frequency Reuse scheme can be efficient solutions to mitigate the interference caused by the deployment of different types of Femtocells in the Macrocells.

All the presented mathematical equations have helped in creating the desired environment to draw a clear comparison in term of vehicular UEs' performance before and after deploying the Fixed and Mobile Femtocells into the Macrocell.

\section{Fixed \& Mobile Femtos scenarios in LTE macrocell}

The following section presents the designed scenarios that have been simulated in MATLAB along with the previous presented mathematical equations in order to create the required environment. Three scenarios have been designed in order to draw a clear comparison between vehicular UEs' performance before and after deploying the Fixed-Femtos and Mobile-Femtos into the LTE Macrocell. This will make it easier to see the impact of the VPL and Path Loss on the performance of those UEs before and after utilising Femtocells technologies in LTE vehicular environments. Hence, the designed scenarios have been classified as the following:

\subsection{Macrocell (eNB) - vehicular UEs scenario}

The first scenario represents the case when the eNB serves the vehicular UEs under high LTE VPL. This scenario works efficiently when the penetration loss and the path-loss are low, but this is not always the case especially when the concern is about the vehicular UEs who are more exposed to high VPL, high path-loss and high interference. It is obvious here that this scenario demonstrates the case of Macrocell before deploying the Femtocells, and when all the links between the UEs and the eNB are Direct links as Fig. 6 shows.

\subsection{Fixed Femtos - vehicular UEs scenario}

The second scenario shown in Fig. 7 represents the case when the Fixed-Femtos are installed in bus stations and railway stations or even outdoor nearer to the threshold of the cell to improve the 


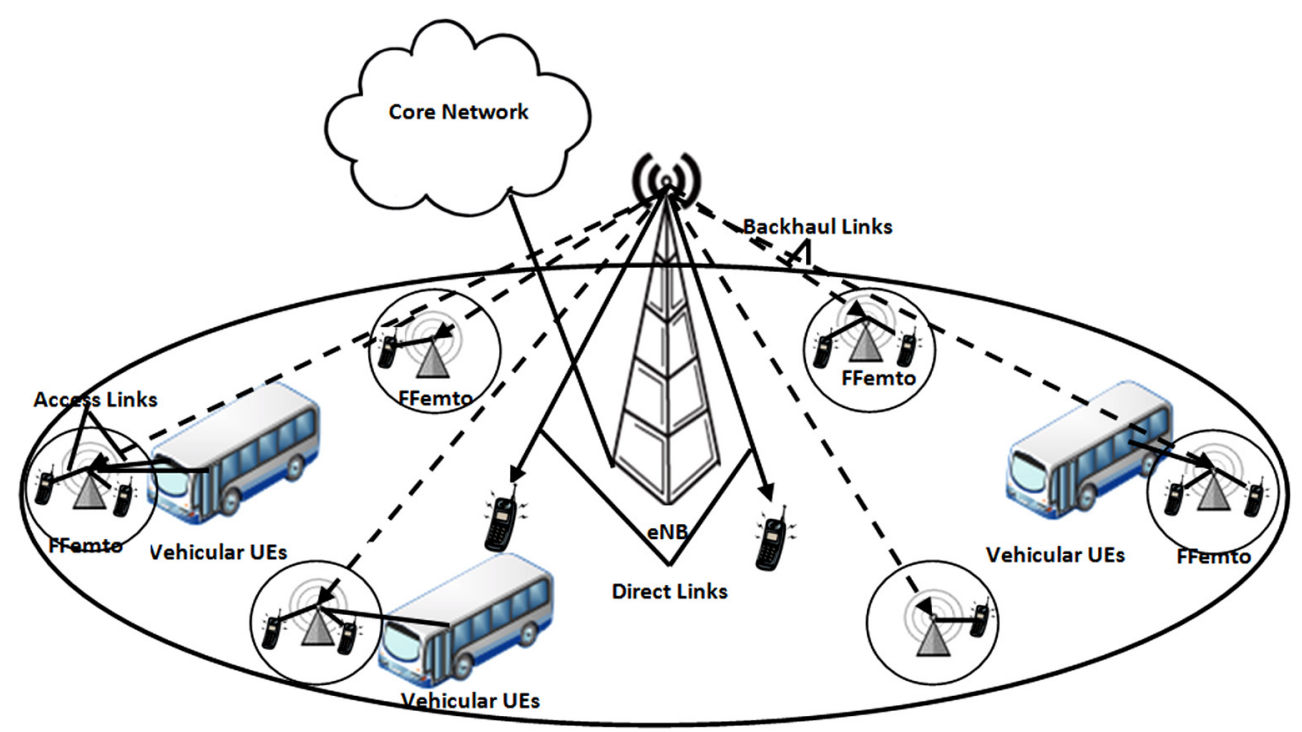

Fig. 7. Vehicular and Mobile UEs served by the Fixed-Femtos.

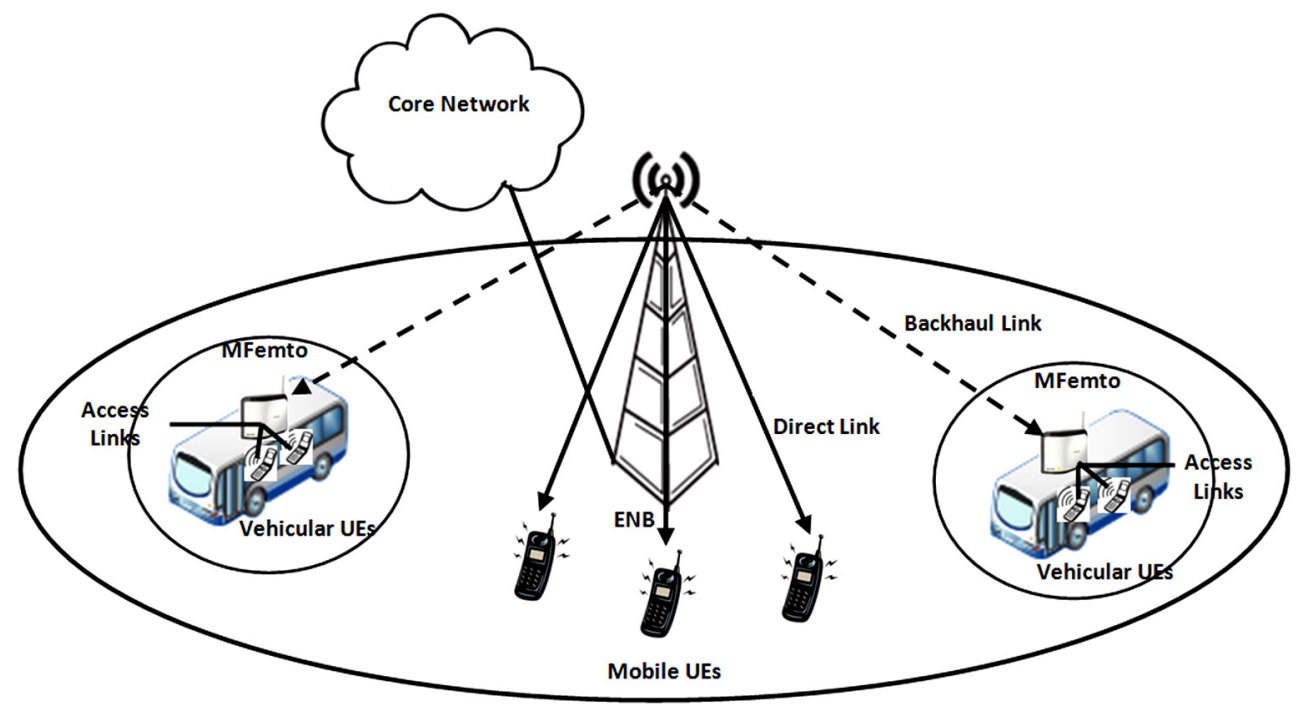

Fig. 8. Vehicular UEs served by Mobile-Femtos.

vehicular UEs performance. In other words, this scenario demonstrates the possibility of serving those vehicular UEs even for a short period of time and study the impact of implementing FixedFemtos to serve vehicular UEs when the VPL is quite high. Deploying these Fixed-Femtos at fixed positions may generate several issues in term of vehicular UEs; e.g. unnecessary number of HOs, high dropped and blocked call probabilities and high outage probability [20].

\subsection{Mobile-Femtos - vehicular UEs scenario}

The third scenario represents the case when the Mobile-Femtos are deployed to serve the vehicular UEs and improve their performance as Fig. 8 shows [22]. These Femtocells can be possibly installed in buses to serve the bus passengers where several criteria are needed to be considered e.g. UEs/Mobile-Femto speed, direction and distance.

\section{Results and discussion}

The performance of vehicular UEs in an LTE network has been evaluated using the dynamic system level simulator, which uses the LTE specification [10]. The Simulator uses the Microcell NLOS path-loss model, which is based on the COST 231 Walfish-Ikegami NLOS model with urban environment. This model is more appropriate when the distance between two BSs is less than $1 \mathrm{~km} \mathrm{[1].}$ The vehicular UEs who have been served by the eNB, Fixed-Femtos and Mobile-Femtos were distributed randomly in the Macrocell, while the Femtocells' coverage has been distributed based on the Microcell NLOS path-loss model. The fast fading model [10] is generated according to the speed of the UEs/Mobile-Femtos and the used transmission mode. The environment uses PF scheduler, as it is more efficient in the case of vehicular environment in order to avoid interference. The directional TS36.942 antenna specification is used for the simulated eNBs with a gain of $15 \mathrm{dBi}$ while omnidirectional antenna is used for the Fixed-Femtos and Mobile-Femtos with a gain of $0 \mathrm{dBi}$. The MIMO is used as a transmission mode in order to have a better throughput and serve more UEs.

As a result, a single base station with three sites ( 3 eNBs) has been considered where three Fixed-Femtos or two Mobile-Femtos have been distributed in each $1 \mathrm{~km}^{2}$. The previous values and distribution have been chosen based on the NLOS Microcell path-loss module in order to mitigate the interference issue as shown in 


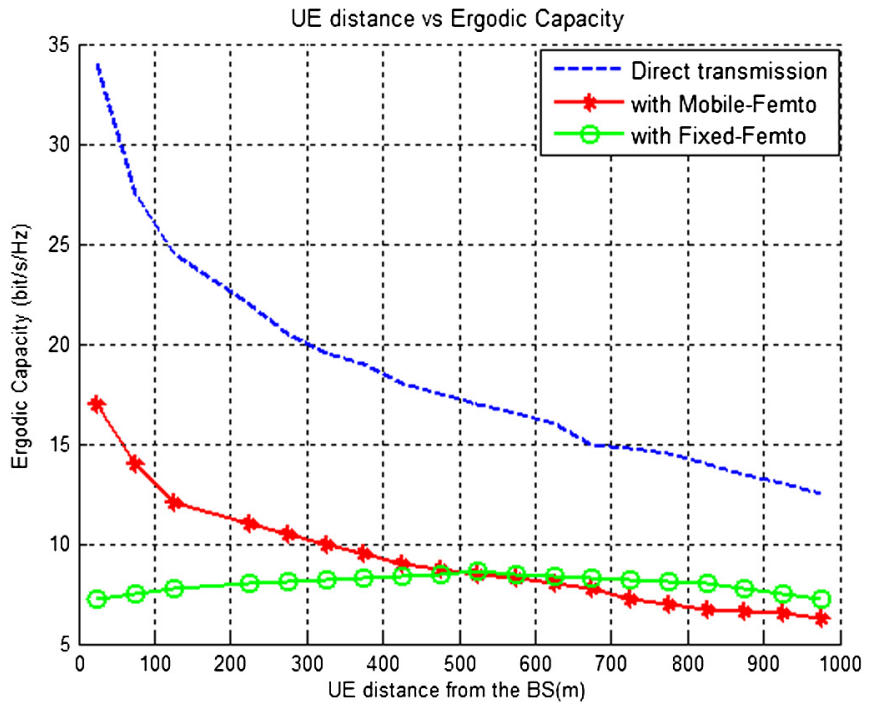

Fig. 9. The ergodic capacity when the $\mathrm{VPL}=0 \mathrm{~dB}$.

[26]. The eNB and Fixed-Femto/Mobile-Femto UEs were assumed to be 40 and 10 respectively in each Macrocell. The LTE frame structure has been considered, which consists of blocks of 12 contiguous subcarriers in the frequency domain and 7 Orthogonal Frequency Division Multiplexing (OFDM) symbols in the time domain. The scheduling period is $1 \mathrm{~ms}$ per each sub-frame. The carrier BW is fixed at $10 \mathrm{MHz}$ with 50 PRBs. A full eNB buffer is considered where there is always buffered data ready for transmission for each node. Both the eNB and the Fixed-Femto/Mobile-Femto transmit data with fixed power per PRB. The transmission power of the eNB and Fixed-Femtos/Mobile-Femtos were assumed to be $46 \mathrm{dBm}$ and $24 \mathrm{dBm}$ respectively based on our previous study in [26]. Furthermore, the speed of the Mobile-Femto and the vehicular UEs in the bus were assumed to range from $3 \mathrm{~km} / \mathrm{h}$ to $160 \mathrm{~km} / \mathrm{h}$ where different VPL scales have been considered in the simulated environment. Besides that, the simulation was running for 100 Transmission Time Interval (TTIs).

\subsection{Ergodic capacity}

The Ergodic capacity of vehicular UEs' links plays an important role in evaluating their performance as it is significantly affected by both the penetration loss and the path-loss. The Ergodic capacity is the maximum rate that reliable communication can achieve by assuming that the communication duration is long enough to experience all channel states. This has helped in evaluating the direct and access links of vehicular UEs and the impact of each on the achieved capacity. Fig. 9, Fig. 10 and Fig. 11 present the simulated Ergodic capacity under different VPL scales.

In Fig. 9 it is obvious that when there is no VPL, the direct transmission always achieves the highest Ergodic capacity. Low VPL means low resistance against the transmitted signal and the signal can pass through easily without facing a dramatic reduction in the signal's power. Even though the Mobile-Femto is seen as a better option (in preference to using the Fixed-Femto) for vehicular UEs, at $500 \mathrm{~m}$ to $1000 \mathrm{~m}$ the Fixed-Femto shows a flat capacity improvement. This is because vehicular UEs are moving close to cell-edges while there is no penetration loss so those UEs can be served by any nearby Fixed-Femto and that will improve their Ergodic capacity. In other words, when the penetration loss is equal to $0 \mathrm{~dB}$ that means does not exist, being served by fixed Femtocells at high distances from the eNB, sounds a better option than using the Mobile-Femto. That is due to the backhaul link variation between the eNB and Mobile-Femto in high path-loss areas, which

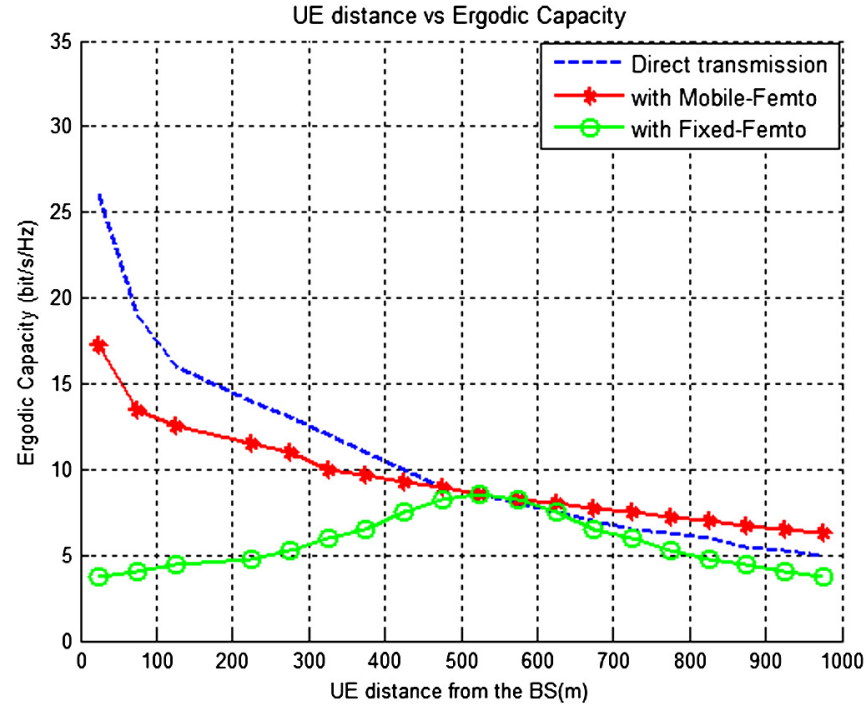

Fig. 10. The ergodic capacity when the $\mathrm{VPL}=25 \mathrm{~dB}$.

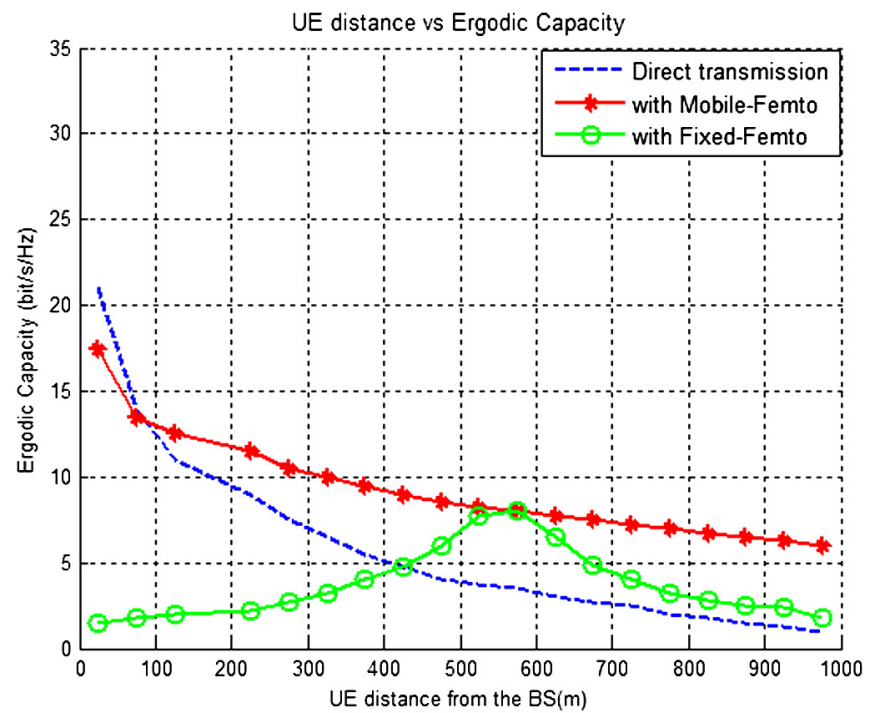

Fig. 11. The ergodic capacity when the VPL $=40 \mathrm{~dB}$.

in turn limits the communication between the two and becomes more obvious in the absence of the VPL. Subsequently, this limits the achieved Ergodic capacity of Mobile-Femto UEs' access links.

Therefore, removing the effect of the VPL from equation (7) has the biggest impact on the achieved Ergodic link capacity between the serving Fixed-Femto and its UEs. Later figures will show obvious degradation in the Ergodic capacity of the Fixed-Femto UEs due to the impact of the increased VPL. This degradation will be a combined with a fluctuation due to the distance variation between the vehicular UEs and the serving Fixed-Femto.

In contrast, Fig. 10 shows the Ergodic capacity when the VPL is equal to $25 \mathrm{~dB}$. It is obvious that at $500 \mathrm{~m}$ distance from the eNB the Mobile-Femto starts to achieve higher capacity in the case of vehicular UEs who are facing high VPL and signal variation. This is because with the increased VPL and path-loss due to the distance from the eNB, the Mobile-Femto in the bus is seen as a better option for the vehicular UEs to be connected to, and to improve their throughput and performance.

In Fig. 11, it is important to state that at a certain stage both of the direct and the Fixed-Femto transmission Ergodic capacity will be poor as the VPL and the path-loss increase due to the distance gap between the vehicular UE and the eNB. 


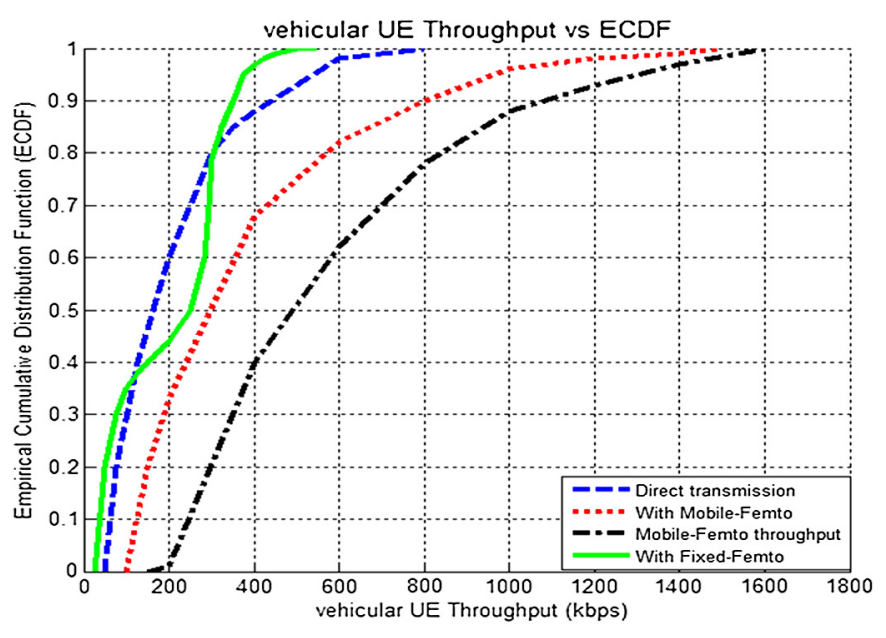

Fig. 12. Vehicular UEs throughput at $\mathrm{VPL}=25 \mathrm{Db}$.

At this stage, deploying Mobile-Femtos inside buses will be the ideal solution to overcome the signal reduction with both increased distance and VPL for vehicular UEs. Moreover, at almost $440 \mathrm{~m}$ distance between the UE and eNB, the Fixed-Femto starts to achieve higher Ergodic capacity - its peak - than the eNB as those vehicular UEs are closer to the Fixed-Femto BS than the eNB. At this point the vehicular UEs experience very high VPL, distance gap and weak signal from the eNB. Therefore, the option for those vehicular UEs is to connect to any nearby Fixed-Femto even for few moments just to maintain the signal connection. However, the capacity drops again as long the distance increases between the Fixed-Femto and the vehicular UEs.

After reviewing the results of the vehicular UEs links Ergodic capacity, it is important now to consider the other performance evaluation elements like the throughput, spectral efficiency and SINR.

\subsection{Throughput}

This study has shown that when a Mobile-Femto is deployed, the number of scheduled vehicular UEs increases. As a result, the throughput of those vehicular UEs improves. This is due to the fact that Mobile-Femto can reach areas which the Fixed-Femto cannot, and this confers an advantage for the Mobile-Femto over the FixedFemto. Additionally, the penetration loss inside vehicles plays an important role in the throughput degradation as eNB and FixedFemto signals have to penetrate the chassis of the vehicles in order to reach the vehicular UEs.

The vehicular UEs and Mobile-Femto throughput in respect to the Empirical Cumulative Distribution Function (ECDF) is shown in Fig. 12. The results show a comparison between the vehicular UE throughput before and after implementing the Femtocells into the Macrocell. Clearly, implementing the Fixed-Femto into the Macrocell does not improve the vehicular UEs throughput due to the VPL and path-loss issues.

However, at a certain distance, the Fixed-Femto vehicular UEs start to achieve a slight higher throughput than the eNB vehicular UEs. This is because the vehicular UEs are moving and are at varying distances from a nearby Fixed-Femto, especially when the distance gap increases between the vehicular UEs and the eNB this is what the intersection areas have shown. In contrast, the throughput drops again as the VPL and distance gap (path-loss) increase between the Fixed-Femto and vehicular UE. Therefore, deploying the Mobile-Femto in the Macrocell shows an improvement in the vehicular UEs throughput, as $80 \%$ of the vehicular UEs throughput increased by 300 Kbps.

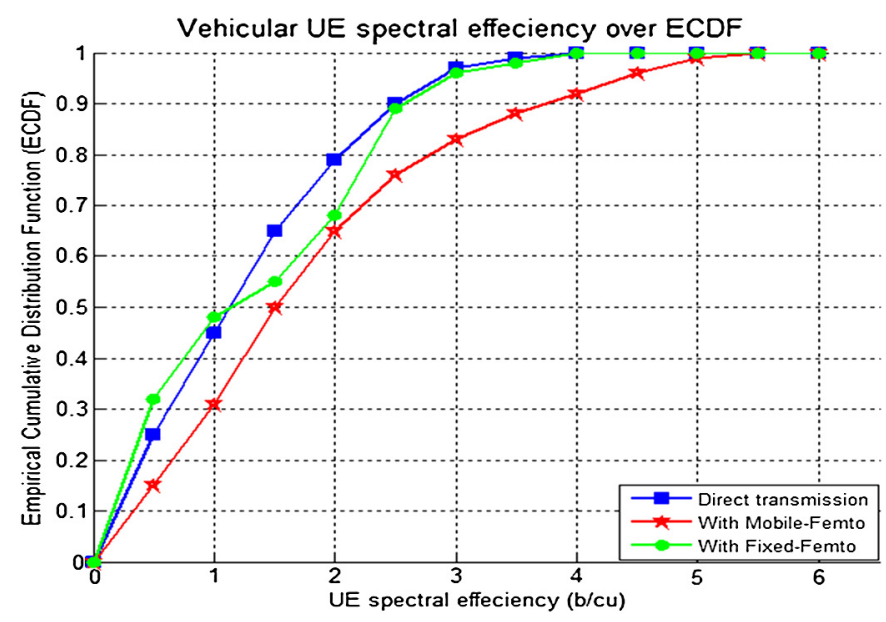

Fig. 13. Spectral efficiency of vehicular UEs at VPL $=25 \mathrm{~dB}$.

Furthermore, the black curve shows that the Mobile-Femtos themselves have a higher throughput around $500 \mathrm{Kbps}$ due to the additional gain in the received SINR on the backhaul link. This has improved the transmitted signal between the eNB and the MobileFemto, thus the achieved throughput, and this is based on study [5]. This gain can be achieved by using a highly directional antenna pattern in the eNB and directing it towards the positioned MobileFemto antenna. Also, it is to be mentioned that the throughput has been improved after reducing the interference issue. This has been achieved by specifying the Mobile-Femto paths based on the used NLOS Microcell path-loss module [26].

\subsection{Spectral efficiency}

The spectral efficiency is highly affected by VPL, path-loss and interferences together with other factors like the femtocells transmission power [23], the femtocells distributions over distance [25] and finally the speed of the developed Mobile-Femto technology [24]. Addressing these issues, Fig. 13 represents a comparison between the spectral efficiency in respect to the ECDF of vehicular $\mathrm{UE}$ in the case of direct transmission from the eNB and in the case of implementing the Fixed-Femto and Mobile-Femto in the Macrocell. There was an obvious improvement in the spectral efficiency of the vehicular UE after implementing the Mobile-Femto rather than the Fixed-Femto.

This is for two reasons. Firstly, the UEs are in vehicles (in this case, buses) and moving from one place to another, which makes it hard for them to establish a long duration connection with the nearby Fixed-Femto, unless those UEs have stopped for few minutes close to a Fixed-Femto that has been deployed in a nearby bus station. This explains why the figure shows a slight improvement in the case of vehicular UEs spectral efficiency when they have been attached to the Fixed-Femto compared with the direct transmission UEs. As the distance between the vehicular UEs and the eNB increases, the Fixed-Femto starts to look a better option than relying on the eNB to provide the connection for them. This will be further explained in the following paragraph.

Secondly, the high VPL (25 dB in the case of vehicular UEs) plays an important role in the poor spectral efficiency of direct transmission as well as the Fixed-Femto UEs transmission. As can be seen in Fig. 13, 90\% of the vehicular UEs have enjoyed a spectral efficiency around $3.7 \mathrm{bit} / \mathrm{cu}$ when they are connected to the Mobile-Femto, versus $2.5 \mathrm{bit} / \mathrm{cu}$ in the case of direct and FixedFemto transmissions. However, there is a slight improvement in the case of Fixed-Femto UEs spectral efficiency over the direct transmission UEs. This is because, as the distance between the vehicular UEs and the eNB increases, the Fixed-Femto starts to look 


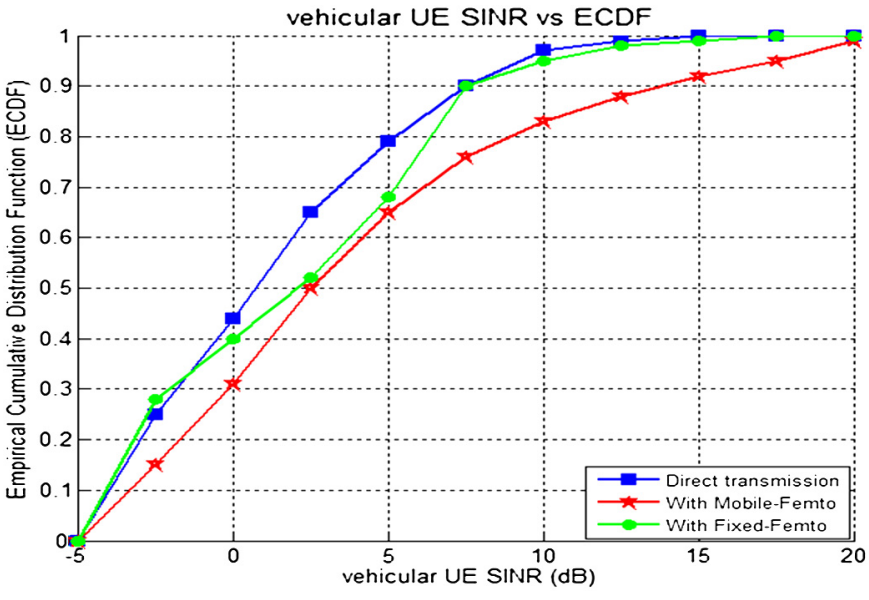

Fig. 14. SINR of vehicular UEs at VPL $=25 \mathrm{~dB}$.

as a better option than relying on the eNB to provide the connection for them. Secondly, the high penetration loss that is $25 \mathrm{~dB}$ in the case of vehicular UEs plays an important role in the poor spectral efficiency of direct transmission with the increased distance. This has led to a fluctuated improvement in the spectral efficiency of the Fixed-Femto UEs over the direct UEs which can be noticed through the intersection areas between the two spectral efficiency lines until this improvement becomes stable.

\subsection{SINR}

Added to the spectral efficiency and throughput, the SINR of vehicular UEs plays an important role in measuring the UEs performance. The SINR reflects the signal strength especially for those UEs who are suffering from high VPL and path-loss. Fig. 14 presents the vehicular UEs SINR before and after implementing Femtocells into the Macrocell. The results show that $80 \%$ of the vehicular UEs have increased their SINR by $4 \mathrm{~dB}$, and as a result, implementing the Mobile-Femto into the Macrocell has been a reasonable solution to overcome the signal degradation.

However, the vehicular UEs SINR served by the Fixed-Femtos showed slight improvement at a distance between the vehicular UEs and the eNB of more than $500 \mathrm{~m}$. Therefore, the vehicular UEs will try to establish a connection to maintain their signal with any nearby Fixed-Femto even for a short period of time. As a result, deploying Mobile-Femtos as well as Fixed-Femtos in the Macrocell can be seen as a major development for next generation networks to provide Internet in buses and along bus routes when the penetration and path losses are very high.

\section{Conclusion}

This paper has shown the importance of having a mobile base station to serve vehicular UEs inside public transportation. As shown in the literature, vehicular UEs are very exposed to high VPL, path-loss, interference and performance degradation. Therefore, Mobile-Femto technology with its processes has been proposed as a solution to improve the cell-edge vehicular UEs performance. The presented mathematical equations have been simulated together with the proposed scenarios to create a comprehensive comparison between the eNB, Fixed-Femto and MobileFemto assisted transmissions. This comparison has been evaluated by comparing the achieved performance in terms of vehicular UEs Link Ergodic capacity, throughput, spectral efficiency and SINR. All the simulated results have shown an improvement in the vehicular UEs performance after implementing the Mobile-Femto in public transportation compared to other transmissions. This improvement has been noticed not only in the signal strength inside public transportation but in the achieved throughput of vehicular UEs. It was found that $80 \%$ of vehicular UEs throughput was improved by $300 \mathrm{Kbps}$ over the direct transmission from the eNB.

\section{Acknowledgements}

The authors would like to thank all those who contributed to the completion and success of this work. Also, the Science and Technology department at Middlesex University played a significant role in supporting and backing this work at all stages of the study.

\section{References}

[1] 3GPP Specification, Physical layer aspects for E-UTRA, Technical report. Accessed on 12 November 2011, accessed at http://www.3gpp.org/ftp/Specs/ html-info/25814.htm.

[2] B. Ai, R. He, Z. Zhong, K. Guan, B. Chen, P. Liu, Y. Li, Radio wave propagation scene partitioning for high-speed rails, Int. J. Antennas Propag. (2012) 815232, 7 pages.

[3] M. Andrews, C. Veronigue, A. Feki, P. Gupta, Autonomous spectrum sharing for mixed LTE Femto and macro cells deployments, in: Proc. of IEEE INFOCOM, 2010.

[4] T. Beniero, S. Redana, J. Hamalainen, B. Raaf, Effect of relaying on coverage in 3GPP LTE-advanced, in: IEEE Vehicular Technology Conference, VTC, 2009.

[5] O. Bulakci, On Bakhuling of Relay Enhanced Networks in LTE-Advanced, Department of Communication and Networking, Alto University, Finland, 2010.

[6] M.Z. Chowdhury, S.Q. Lee, B.H. Ru, N. Park, Y.M. Jang, Service quality improvement of mobile users in vehicular environment by Mobile Femtocell network deployment, in: ICT Convergence (ICIT) International Conference, 2011.

[7] T.M. Cover, J.M. Thomas, Elements of Information Theory, September 2006.

[8] M.H. Habaebi, J. Chebil, A.G. Sakkaf, T.H. Dahawi, Comparison between scheduling techniques in Long Term Evolution, IIUM Eng. J. 14 (1) (2013).

[9] F. Haider, M. Dianati, R. Tafazolli, A simulation Based Study of Mobile Femtocell Assisted LTE Networks, Center for Communication System Research, University of Surrey, UK, 2011.

[10] J.C. Ikuno, M. Wrulich, Dimensioning Vienna LTE Simulators System Level Simulator Documentation, v1.8r1375, Instit. of Comm. and Radio-Frequency Engg., Vienna Univ. of Tech., Austria Gusshausstrasse 25/389, A-1040 Vienna, Austria, 2014.

[11] S. Jangsher, V.O.K. Li, Resource allocation in cellular networks employing Mobile Femtocells with deterministic mobility, in: IEEE Wireless Communications and Networking Conference, WCNC, 2013.

[12] I. Kostanic, C. Hall, J. McCarthy, Measurements of the Vehicle Penetration Loss Characteristics at $800 \mathrm{MHz}$, TEC CELLULAR, Inc., 7619 Emerald Drive, West Melbourne, FL 32904, 1998.

[13] P. Kulkarni, W.H. Chin, T. Farnham, Radio resource consideration for LTE, ACM SIGCOMM Comput. Commun. Rev. 40 (1) (January 2010).

[14] T. Mimura, K. Yamamoto, A. Iwata, A. Nishio, M. Morikura, Multi-operator mobile-relaying: effect of shared spectrum allocation, in: IEEE 23th International Conference on Personal Indoor and Mobile Radio Communications, PIMRC, 2012.

[15] R. Raheem, A. Lasebae, M. Aiash, J. Loo, From fixed to Mobile Femtocells in LTE systems: issues and challenges, in: Proc. of the IEEE 2nd International Conference on Future Generation Communication Technologies, FGCT-2013, London, United Kingdom, 2013.

[16] S. Scott, J. Leinonen, P. Pirinen, J. Vihriala, V.V. Phan, M. Latva-aho, A Cooperative Moving Relay Node System Deployment In A High Speed Train, Centre for Wireless Commun., Univ. of Oulu, Oulu, Finland, 2013.

[17] Y. Sui, J. Vihriala, A. Papadogiannis, M. Sternad, Moving Cells: A promising Solution to Boost Performance for Vehicular Users, Chalmers University of Technology, 2013.

[18] S. Tombaz, M. Usman, J. Zander, Energy efficiency improvements through heterogeneous networks in diverse traffic distribution scenarios, in: Proc. of the 6th IEEE International Conference on Communications and Networking in China, CHINACOM, Harbin, China, 2011.

[19] Ofcom, Options for improving in-building mobile coverage, White Paper. Accessed on 21 October 2017, available at https://www.ofcom.org.uk/_data/ assets/pdf_file/0015/63006/final_report.pdf?lang=en.

[20] R. Raheem, A. Lasebae, M. Aiash, J. Loo, Mobility Management in LTE/Mobile Femtocell Networks: Outage Probability and Drop/Block Calls Probability, ERPSS2015, Dubai, United Arab Emirates, 2015.

[21] P. Rysavy, Challenges and Considrations in Defining Spectrum Efficiency, IEEE Member, 2014.

[22] R. Raheem, A. Lasebae, M. Aiash, J. Loo, Performance evaluation of mobile users served by fixed and Mobile Femtocells in LTE networks, in: Proc. of the International Journal of Network Technology, 2016. 
[23] Tweet 4 Tutorial, Tweet for LTE (Long Term Evolution) and scripting tutorials: Inter-Cell Interference Coordination (ICIC), Tutorial. Accessed on 20 February 2016, available at http://tweet4tutorial.com.

[24] K. Dimou, M. Wang, Y. Yang, M. Kazmi, A. Larmo, J. Pettersson, W. Muller, Y. Timner, Handover within 3GPP LTE: design principles and performance, in: IEEE 70th Vehicular Technology Conference, VTC, 2009.

[25] S. Olariu, M.C. Weigle, Vehicular Networks: From Theory to Practice, Chapman \& Hall/CRC Comput. Inf. Sci. Ser., 2007.

[26] R. Raheem, A. Lasebae, M. Aiash, J. Loo, Interference management for cochannel Mobile Femtocells technology in LTE networks, in: Proc. of the 12th International Conference on Intelligent Environment, IE'16, United Kingdom, 2016.
[27] S. Xing, P. Ghosal, S. Barua, R. Subramanian, K. Sandrasegaran, System leve simulation for two tier Macro-Femto cellular networks, Int. J. Wirel. Mob. Netw. 6 (6) (2014)

[28] E. Tanghe, W. Joseph, L. Verloock, L. Martens, Evaluation of vehicle penetration loss at wireless communication frequencies, IEEE Trans. Veh. Technol. 57 (4) (2008) 2036-2041.

[29] BeFemto Project Consortium, Broadband evolved FEMTO networks. Accessed on 21 January 2017, available at www.ict-befemto.eu.

[30] C. Cox, An Introduction to LTE: LTE, LTE-Advanced, SAE and 4G Mobile Communications, John Wiley \& Sons Publisher, 2012. 